\title{
OPEN Comparative mitochondrial genome analysis reveals intron dynamics and gene rearrangements in two Trametes species
}

Cheng Chen ${ }^{1,4}$, Qiang $\mathrm{Li}^{2,4}$, Rongtao $\mathrm{Fu}^{1}$, Jian Wang ${ }^{1}$, Guangmin Deng ${ }^{3}$, Xiaojuan $\mathrm{Chen}^{1}$ \& Daihua Lu' ${ }^{1,3 \otimes}$

Trametes species are efficient wood decomposers that are widespread throughout the world. Mitogenomes have been widely used to understand the phylogeny and evolution of fungi. Up to now, two mitogenomes from the Trametes genus have been revealed. In the present study, the complete mitogenomes of two novel Trametes species, Trametes versicolor and $T$. coccinea, were assembled and compared with other Polyporales mitogenomes. Both species contained circular DNA molecules, with sizes of $67,318 \mathrm{bp}$ and $99,976 \mathrm{bp}$, respectively. Comparative mitogenomic analysis indicated that the gene number, length and base composition varied between the four Trametes mitogenomes we tested. In addition, all of the core protein coding genes in Trametes species were identified and subjected to purifying selection. The mitogenome of $T$. coccinea contained the largest number of introns among the four Trametes species tested, and introns were considered the main factors contributing to size variations of Polyporales. Several novel introns were detected in the Trametes species we assembled, and introns identified in Polyporales were found to undergo frequent loss/gain events. Large-scale gene rearrangements were detected between closely related Trametes species, including gene inversions, insertions, and migrations. A well-supported phylogenetic tree for 77 Basidiomycetes was obtained based on the combined mitochondrial gene set using 2 phylogenetic inference methods. The results showed that mitochondrial genes are effective molecular markers for understanding the phylogeny of Basidiomycetes. This study is the first to report the mitogenome rearrangement and intron dynamics of Trametes species, which shed light on the evolution of Trametes and other related species.

Trametes species, belonging to Polyporales, Basidiomycota, are widely distributed throughout the world ${ }^{1}$. Trametes species are often distributed on standing dead or fallen hardwood trees ${ }^{2}$. Like other species from the order Polyporales, Trametes species are efficient wood decomposers that cause white rot in colonized wood. Their efficient lignin decomposition ability makes Trametes important decomposers in ecosystems and plays an important role in the natural cycle of earth forest ecosystems. In addition, Trametes species have also been used in ex situ and in situ biosynthesis and bioremediation studies ${ }^{3-6}$. A series of lignin-degrading enzyme systems and genes have been found to be closely related to the excellent degradation ability of Trametes $^{7-9}$, including lignin peroxidases, manganese peroxidases, and laccases. To date, only two mitochondrial genomes (mitogenomes) of Trametes species have been reported ${ }^{10,11}$, including T. cingulata and T. hirsute, and the characterizations and differentiation of mitogenomes in Trametes have not been fully analyzed.

\footnotetext{
${ }^{1}$ Institute of Plant Protection, Sichuan Academy of Agricultural Sciences, Key Laboratory of Integrated Pest Management On Crops in Southwest, Ministry of Agriculture, Chengdu, People's Republic of China. ${ }^{2}$ Key Laboratory of Coarse Cereal Processing, Ministry of Agriculture and Rural Affairs, School of Food and Biological Engineering, Chengdu University, Chengdu, People's Republic of China. ${ }^{3}$ Present address: Sichuan Academy of Agricultural Sciences, 20 \# Jingjusi Rd, Chengdu 610066, Sichuan, People's Republic of China. ${ }^{4}$ These authors contributed equally: Cheng Chen and Qiang Li ${ }^{\bowtie}$ email: Daihualu@126.com
} 


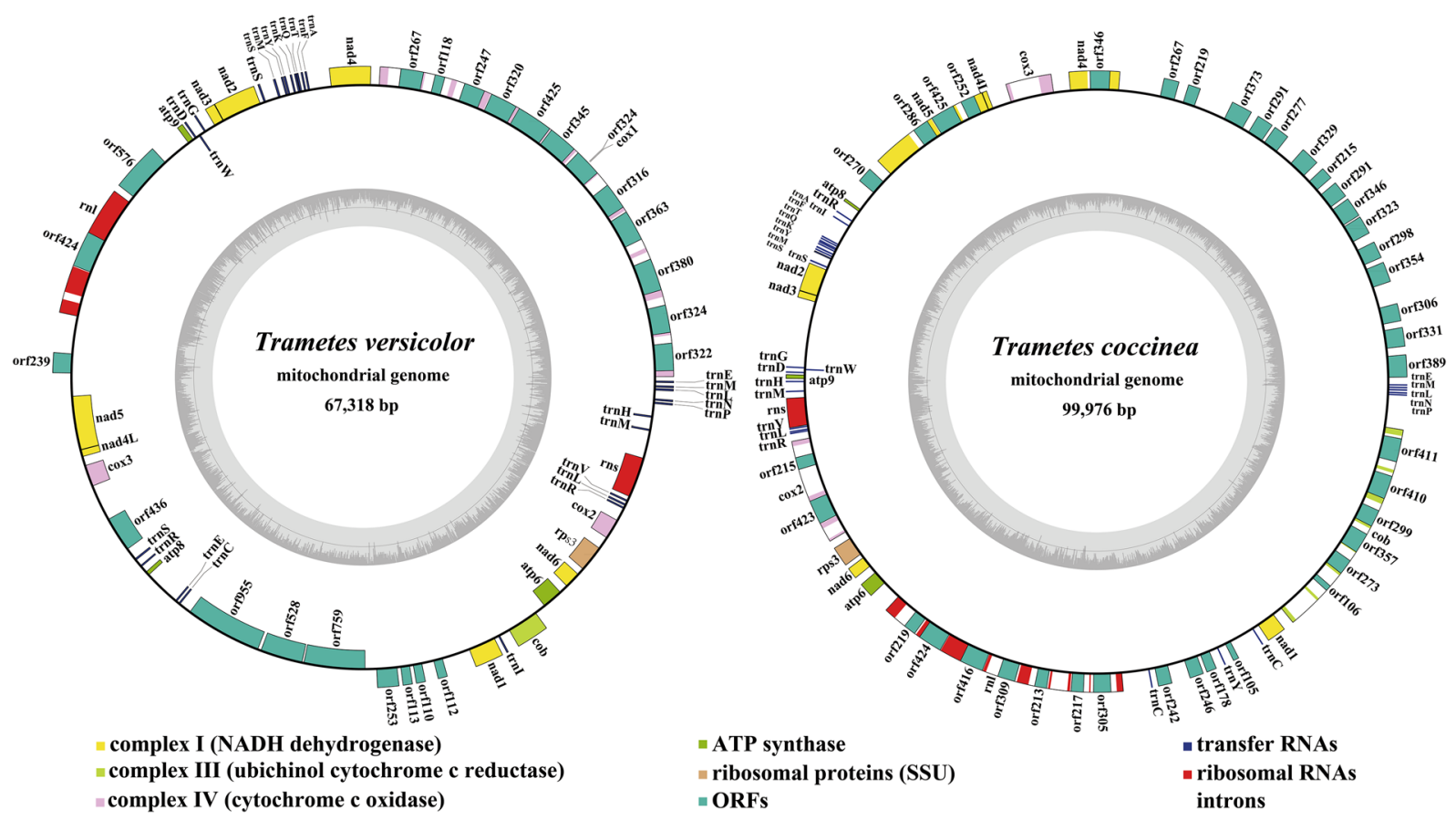

Figure 1. Circular maps of the two Trametes mitogenomes. Genes are represented by different colored blocks. Colored blocks outside each ring indicate that the genes are on the direct strand, while colored blocks within the ring indicates that the genes are located on the reverse strand.

Most eukaryotes contain mitogenomes, which are thought to be derived from Alphaproteobacteria through endosymbiosis ${ }^{12,13}$. The variation or mutation of mitogenomes can affect the growth, metabolism, and development of eukaryotes, leading to their aging and even death ${ }^{14,15}$. In addition, mitogenomes are also an effective tool to understand the evolution and phylogeny of eukaryotes ${ }^{16,17}$. Other characteristics of mitogenomes, such as genome size, intron type, tRNA genes, gene arrangement, and repeat sequences, have also become important references to reflect the evolutionary status of species ${ }^{18-21}$. The size, structure, and content of fungal mitogenomes vary greatly ${ }^{22-24}$, which has made it difficult to obtain complete fungal mitogenomes. When compared with the available fungal nuclear genome, the number of available fungal mitogenomes is very low (https://www.ncbi.nlm. nih.gov/genome/browse\#!/overview/). The rapid development of high-throughput sequencing technologies, such as 454 pyrosequencing, Illumina (Solexa) sequencing, ABI SOLiD sequencing, Oxford Nanopore sequencing, and Pacbio SMRT sequencing, has promoted understanding of fungal mitogenomes.

In the present study, the mitogenomes of two Trametes species, Trametes versicolor and T. coccinea, were assembled and compared. The goals of this study were to: (1) characterize Trametes mitogenomes; (2) reveal the variations or similarities in genome size, structure, and gene content of the Trametes mitogenomes; (3) reveal the dynamic changes of introns in Polyporales mitogenomes; and (4) shed light on the phylogenetic relationships of Trametes in the phylum Basidiomycota based on the combined mitochondrial gene set. The results of this study will promote improved understanding of the origin, evolution, and taxonomy of Trametes species and other related fugal species.

\section{Results}

Features and PCGs of Trametes mitogenomes. The complete mitogenomes of T. versicolor and T. coccinea were circular with sizes of $67,318 \mathrm{bp}$ and $99,976 \mathrm{bp}$, respectively (Fig. 1). The GC contents of the T. versicolor and T. coccinea mitogenomes were $25.44 \%$ and $24.68 \%$, respectively (Table S1). Both the AT skew and GC skew in the T. versicolor mitogenome were positive. The mitogenome of T. coccinea contained negative AT skews and positive GC skews. We detected an entire set of core PCGs in the two Trametes mitogenomes we assembled, including atp6, atp8, atp9, cob, cox1, cox2, cox3, nad1, nad2, nad3, nad4, nad4L, nad5, nad6, and rps3 (Table S2). In addition to these core PCGs, ten and five free-standing PCGs (non-intronic ORFs) were detected in the T. versicolor and T. coccinea mitogenomes, respectively. Several genes encoding DNA polymerase and RNA polymerase were detected in the two Trametes mitogenomes. In addition, the T. versicolor and T. coccinea mitogenomes contained seven and five PCGs with unknown functions, respectively. We also detected 14 and 38 introns in the T. versicolor and T. coccinea mitogenomes, respectively, which harbored 13 and 34 intronic ORFs. Intronic ORFs in the two Trametes mitogenomes mainly encoded LAGLIDADG endonucleases, GIY-YIG endonucleases, and intron-encoded RNA maturase bI4; however, we found two and three intronic ORFs with unknown functions in the T. versicolor and T. coccinea mitogenomes, respectively.

rRNA genes and tRNA genes. Both the T. versicolor and T. coccinea mitogenomes contained two rRNA genes: the small subunit ribosomal RNA (rns) and large subunit ribosomal RNA gene ( $r n l$ ) (Table S2). We 


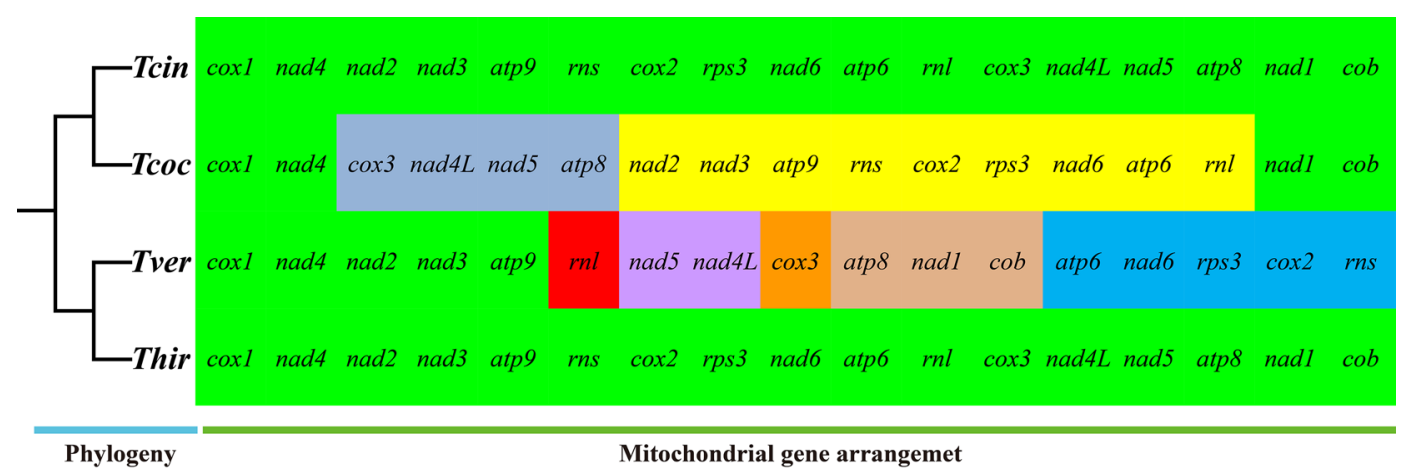

Figure 2. Gene order comparation between 4 Trametes species. All genes are shown in order of occurrence in the mitochondrial genome, starting from coxl. Fifteen core protein coding genes and two rRNA genes were included in the gene arrangement analysis. Genes with a green background indicate that they are conservative in Boletales species. Genes with other colored background indicate that they have gene rearrangements. The phylogenetic positions of 4 Trametes species were established using the Bayesian inference (BI) method and Maximum Likelihood (ML) method based on concatenated mitochondrial genes. Species and NCBI accession number used for gene arrangement analysis in the present study are listed in Supplementary Table S6.

detected two and eight introns in the $\mathrm{rnl}$ genes of T. versicolor and T. coccinea, respectively. The average size of the $r n l$ gene was 3,013 bp, and the average length of $r n s$ genes in the two Trametes mitogenomes was 1,517 bp (excluding intron regions). The T. versicolor mitogenome contained longer $r n l$ and shorter $r n s$ genes than the T. coccinea mitogenome.

The two Trametes mitogenomes we assembled each contained 27 tRNA genes (Table S2) that encoded 20 standard amino acids and ranged in length from 71 to $88 \mathrm{bp}$. The trnS gene contained the largest sizes among all tRNAs detected, mainly because of the large extra arm. Both mitogenomes of $T$. versicolor and $T$. coccinea contained two tRNAs that code for leucine and arginine with different anticodons, as well as three tRNAs encoding serine with different anticodons. The two mitogenomes also contained three tRNAs encoding methionine with the same anticodons. In addition, the T. versicolor mitogenome contained two trnE codons encoding glutamate, while the mitogenome of $T$. coccinea contained two trnC codons encoding cysteine.

Repetitive elements in Trametes mitogenomes. We detected 38 and 45 intragenomic duplications in the T. versicolor and T. coccinea mitogenomes, respectively (Table S3), through BlastN searches of the two Trametes mitogenomes against themselves. The sizes of these intragenomic duplications ranged from 33 to $974 \mathrm{bp}$ in the two Trametes mitogenomes and the pair-wise nucleotide similarities of these duplications were between 66.40 and $100 \%$. The largest repeats were found in the coding regions of orf 425 and orf 424 in the mitogenome of T. coccinea, while the largest repeats in the T. versicolor mitogenome were observed in the intergenic regions between $\operatorname{nad} 5$ and $\operatorname{orf} 270$, as well as between atp 8 and trnR, with each repeating sequence being $240 \mathrm{bp}$ long. Repeat sequences identified by BlastN searches accounted for $4.45 \%$ and $8.29 \%$ of the T. versicolor and T. coccinea mitogenomes, respectively. We also identified 33 and 7 tandem repeats in the mitogenomes of T. versicolor and T. coccinea, respectively (Table S4), using Tandem Repeat Finder. The longest tandem repeat sequence, which had a size of $121 \mathrm{bp}$, was observed in the intergenic region between orf110 and orf112 in the mitogenome of T. versicolor. Tandem repeats accounted for $2.39 \%$ and $0.27 \%$ of the T. versicolor and T. coccinea mitogenomes, respectively.

We also conducted BlastN searches of the two Trametes mitogenomes against their published nuclear genomes to determine whether any gene fragments were naturally transferred between the mitochondrial and nuclear genomes (Table S5). We detected one and four aligned fragments between the mitochondrial and nuclear genomes of T. versicolor and T. coccinea, respectively. These aligned fragments ranged from 45 to $173 \mathrm{bp}$, with pair-wise nucleotide similarities ranging from $90.75 \%$ to $100 \%$. The largest aligned fragment was observed in the intergenic region between $\operatorname{trn} C$ and orf 242 in the T. coccinea mitogenome. A total of $45 \mathrm{bp}$ and $504 \mathrm{bp}$ aligned fragments were detected in the T. versicolor and T. coccinea mitogenomes, respectively, indicating potential gene segment transfering events between mitochondrial and nuclear genomes may have occurred in the evolution of Trametes.

Mitochondrial gene rearrangement in Trametes species. We compared the arrangements of 15 core PCGs and 2 rRNA genes in the 4 Trametes mitogenomes reported in the present study (Fig. 2). Trametes cingulata and T. hirsuta were found to have an identical gene arrangement. We detected large-scale gene rearrangements in mitogenomes of T. versicolor and T. coccinea compared with the identical gene arrangement in $T$. cingulata and T. hirsute. These gene rearrangements included gene migrations, inversions, and insertions.

Mauve $^{25}$ revealed the presence of 10 homologous regions between the 4 Trametes species (Fig. 3), with different Trametes species found to contain various types and numbers of homologous regions. The T. hirsuta and T. versicolor mitogenomes contained additional homologous regions I and J, which involved the intergenic regions between neighboring genes atp 9 and orf576 and between atp 8 and trnE, respectively. Collinearity analysis 


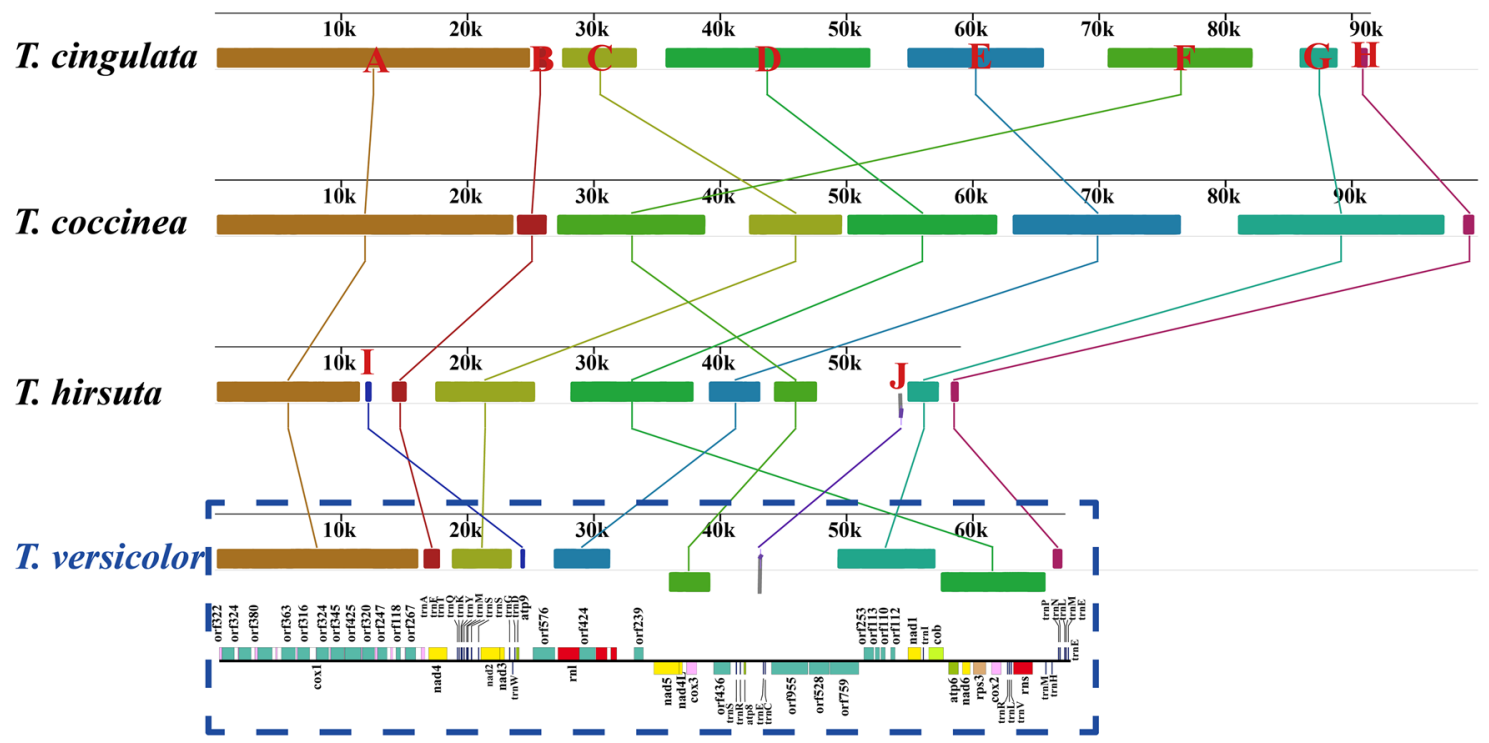

Figure 3. Mitochondrial gene collinearity analysis of 4 Trametes species using Mauve v2.4.0. Color blocks of the same color represent homologous regions between different mitogenomes. The schematic diagram of the mitogenome of Trametes versicolor is shown at the bottom of the picture.

indicated that the mitogenomes of T. cingulata and T. hirsuta were highly collinear, and that the T. versicolor and T. coccinea mitogenomes rearranged in homologous regions $\mathrm{C}, \mathrm{F}$, and $\mathrm{D}$. The results indicated that the mitogenomes of Trametes underwent gene rearrangements in evolution.

Variation, genetic distance, and evolutionary rates of core PCGs. Seven of the 15 core PCGs detected (cox2, nad2, nad3, nad4, nad5, nad6, and rps3) varied in length between the 4 Trametes mitogenomes tested (Fig. 4). The nad5 gene had the largest length variations between Trametes species, while the largest nad5 gene was observed in T. hirsute. All of the 15 core PCGs except atp6, cox2, and nad4L had GC contents that varied between Trametes species. Among the 15 core PCGs detected, the atp 9 gene contained the highest GC content and the atp 8 gene contained the lowest. GC skews of the 15 core PCGs varied, with most showing positive GC skews.

The largest K2P genetic distance between Trametes species was observed in the rps 3 gene, followed by nad3, indicating the two genes differentiated greatly during evolution (Fig. 5). The atp 8 and nad $4 L$ genes exhibited the lowest mean K2P genetic distance between the four Trametes mitogenomes, indicating that they were highly conserved. The rps3 gene exhibited the highest mean non-synonymous substitution $(\mathrm{Ka})$ rate, whereas the nad4L gene had the lowest $K a$ mean value among the 15 core PCGs detected. We also found that the nad 3 gene had the highest mean synonymous substitution rate $(K s)$, whereas the atp 8 gene exhibited the lowest $K s$ mean value among the 15 core PCGs detected. The $\mathrm{Ka} / \mathrm{Ks}$ values for all core PCGs were less than 1 , indicating that the core genes in Trametes species were subjected to pressure of purifying selection.

Intron dynamics of cox 1 gene in Polyporales species. Pearson correlation analysis showed that there was a high correlation coefficient between the number of introns and the size of the mitogenome in the order Polyporales (Fig. 6). The dynamics of introns in Polyporales had a significant effect on Polyporales mitogenome size. The cox1 gene was found to be the largest host gene of introns in Basidiomycota ${ }^{26,27}$. In the present study, introns of Polyporales cox 1 genes were classified into different position classes (Pcls) using the cox 1 gene of Ganoderma calidophilum as a reference ${ }^{22}$, and introns belonging to the same Pcls were considered homologous introns. Overall, 153 introns were detected in the 14 cox 1 genes of the order Polyporales, and the number of introns in each cox 1 gene varied between 6 and 15, with an average of 11 . Five of the 153 introns belonged to the group II, and the other introns belonged to the group I.

Overall, 32 Pcls were detected in the 14 Polyporales species (Fig. 7), with P706, P807, and P1305 being the most common intron types, occurring in 11 of the 14 Polyporales species. These commonly distributed introns may have been obtained from the common ancestor of Polyporales species. Seven of the 32 Pcls (P218, P309, P480, P726, P894, P941, and P1114) were only detected in one of the 14 Polyporales species. The rare introns in Polyporales, such as the P480 and P941, were also detected in distantly related species, such as Armillaria sinapina $^{28}$ and Agaricus bisporus ${ }^{29}$ from the order Agaricales. These results indicated potential horizontal gene transfer events occurred during evolution. However, P218, P309, P276, P894, and P1114 were only distributed in Polyporales, and no homologous introns were found in other Basidiomycota species; accordingly, the origins of these rare introns need to be further investigated. P1107 was the most commonly distributed intron type in the four Trametes species we tested and was present in all four Trametes species. We detected two novel introns in our newly sequenced T. coccinea species, indicating diverse intron types in Trametes species. The varied 

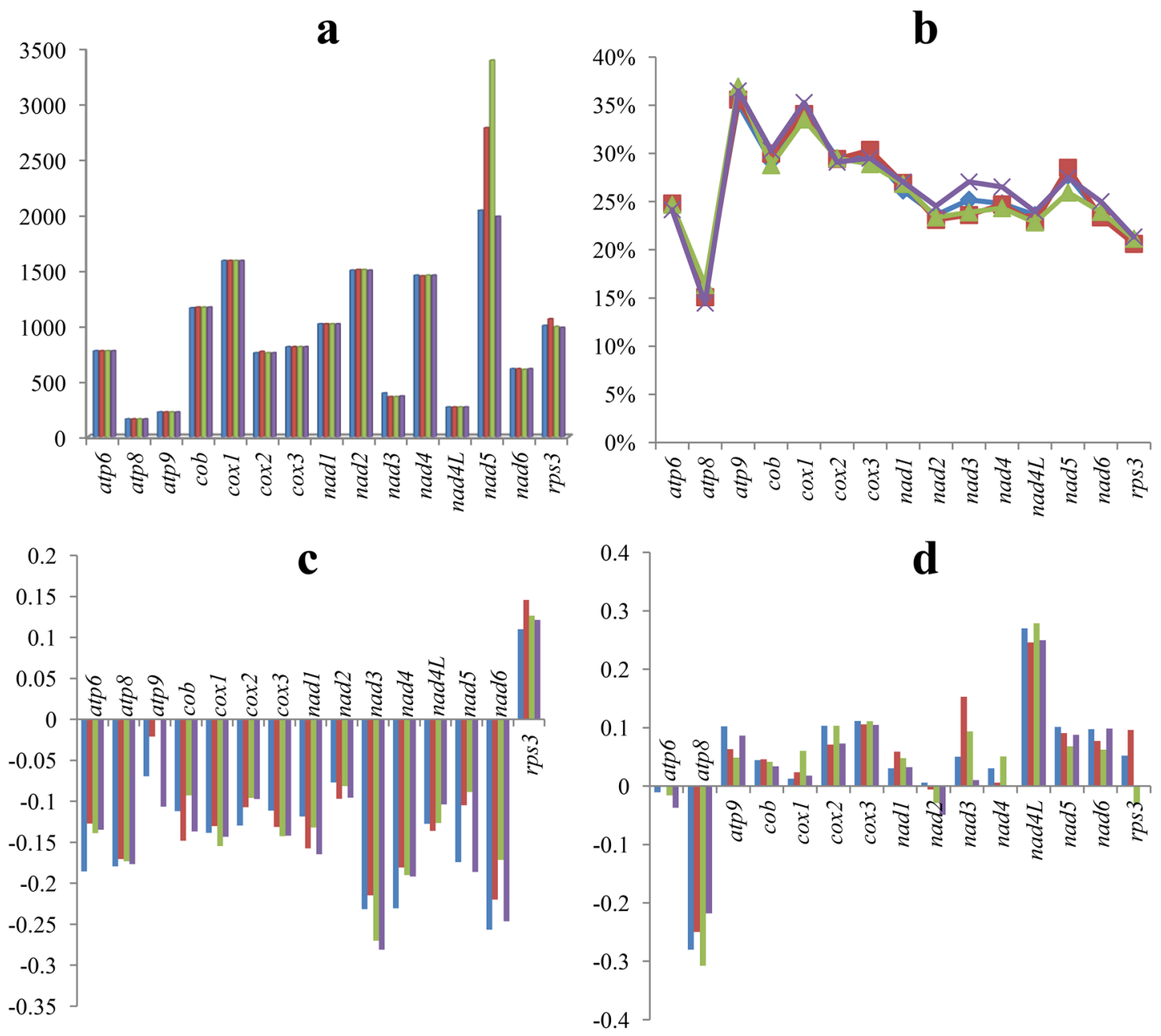

\section{T. cingulata}

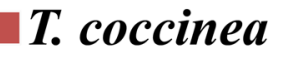

T. hirsuta

T. versicolor

Figure 4. Variation in the length and base composition of each of 15 protein-coding genes (PCGs) between four Trametes mitogenomes. (a) PCG length variation; (b) GC content of the PCGs; (c) AT skew; (d) GC skew.

classes and number of introns in Trametes species indicated that intron gain/loss occurred during evolution of the Trametes mitogenome.

Comparative mitogenome analysis and phylogenetic analysis. The $99.976 \mathrm{~kb}$ mitogenome of $T$. coccinea was the largest mitogenome among the four Trametes species reported ${ }^{10,11}$ (Table S1). When compared with other Polyporales mitogenomes reported, the Trametes mitogenomes had medium sizes. The GC contents of the Polyporales species varied between 24.0 and 36.3\%, with an average of $26.8 \%$. Most Polyporales we detected contained positive AT and GC skews, indicating preferences for A and G bases in the leading strand of Polyporales mitogenomes. All Polyporales mitogenomes tested contained an entire set of core PCGs and a variety of free-standing ORFs. The mitogenome of T. coccinea contained the most introns among the 14 Polyporales species tested. Additionally, the highest number of intronic ORFs was detected in the mitogenome of Phlebia radiata ${ }^{30}$, which contributed to the P. radiata mitogenome becoming the largest mitogenome among the 14 Polyporales species we tested. All 14 Polyporales mitogenomes contained two rRNA genes, and the number of tRNA genes in the 14 Polyporales species ranged from 25 to 29.

An identical and well-supported phylogenetic tree was obtained using the maximum likelihood (ML) and Bayesian inference (BI) methods based on the combined mitochondrial gene set (15 core PCGs) (Fig. 8). All major clades within the phylogenetic tree were found to have good support values ( $\mathrm{BS} \geq 99 ; \mathrm{BPP} \geq 0.96$ ). Overall, 77 Basidiomycota species were included in the phylogenetic analysis, and these were divided into 13 major clades corresponding to the orders Agaricales, Boletales, Russulales, Polyporales, Hymenochaetales, Gomphales, Cantharellales, Pucciniales, Tremellales, Trichosporonales, Microbotryales, Sporidiobolales, Microstromatales, Ustilaginales, and Tilletiales (Table S6). T. coccinea was a sister species to T. cingulata and T. versicolor was closely related to T. hirsute. 

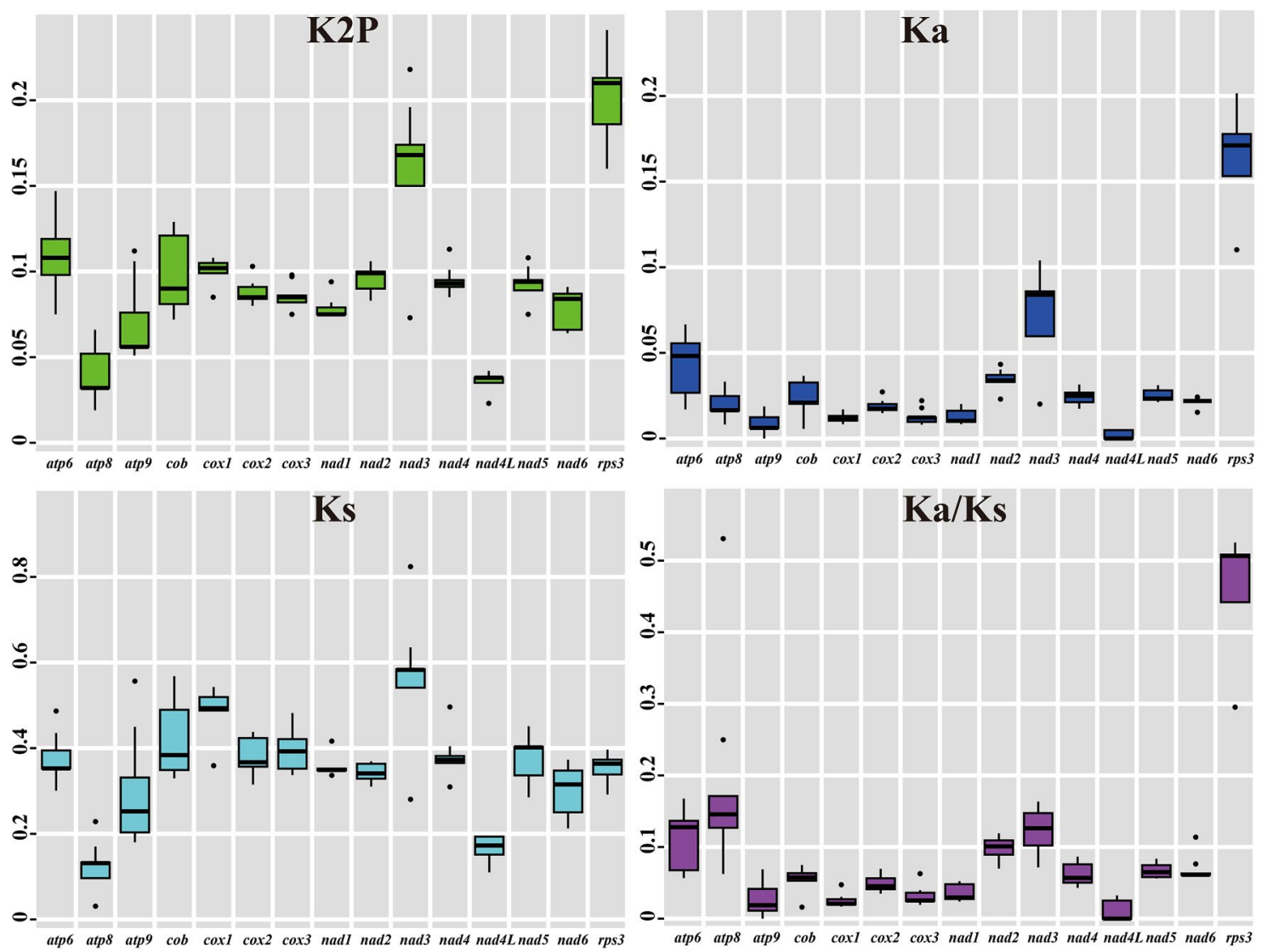

Figure 5. Genetic analysis of 15 protein coding genes conserved in the four Trametes mitogenomes. K2P, the Kimura-2-parameter distance; Ka, the mean number of nonsynonymous substitutions per nonsynonymous site; Ks, the mean number of synonymous substitutions per synonymous site.

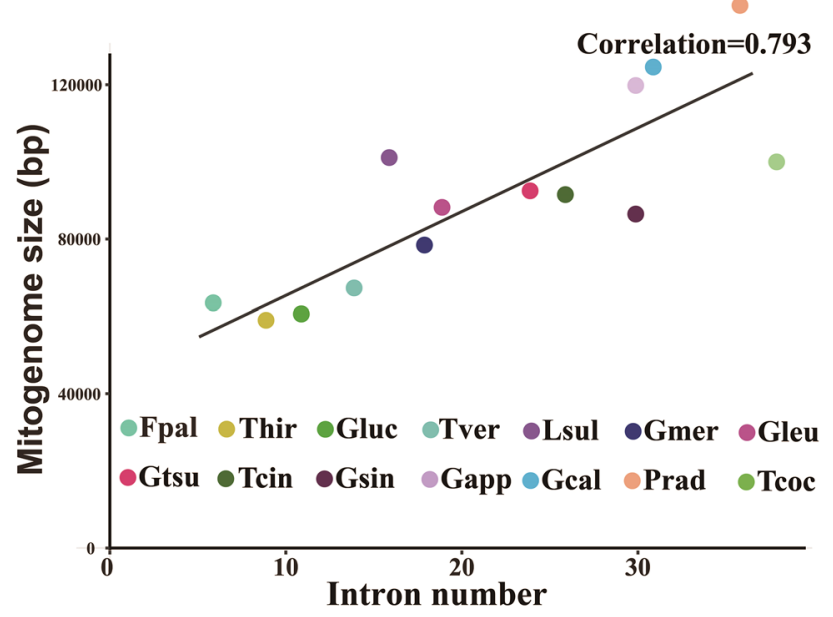

Figure 6. Pearson correlation analysis between the number of intron and mitogenome sizes of 14 Polyporales species. Species and NCBI accession numbers for genomes used in the phylogenetic analysis are provided in Supplementary Table S1.

\section{Discussion}

Size and content variations between Trametes mitogenomes. In this study, two mitogenomes of the genus Trametes, T. coccinea and T. versicolor, were newly assembled. Comparative mitogenomic analysis showed that the novel mitogenome of $T$. coccinea was largest among the four Trametes mitogenomes reported ${ }^{10,11}$. Introns were considered the main factors contributing to the size expansion of the T. coccinea mitogenome, and 


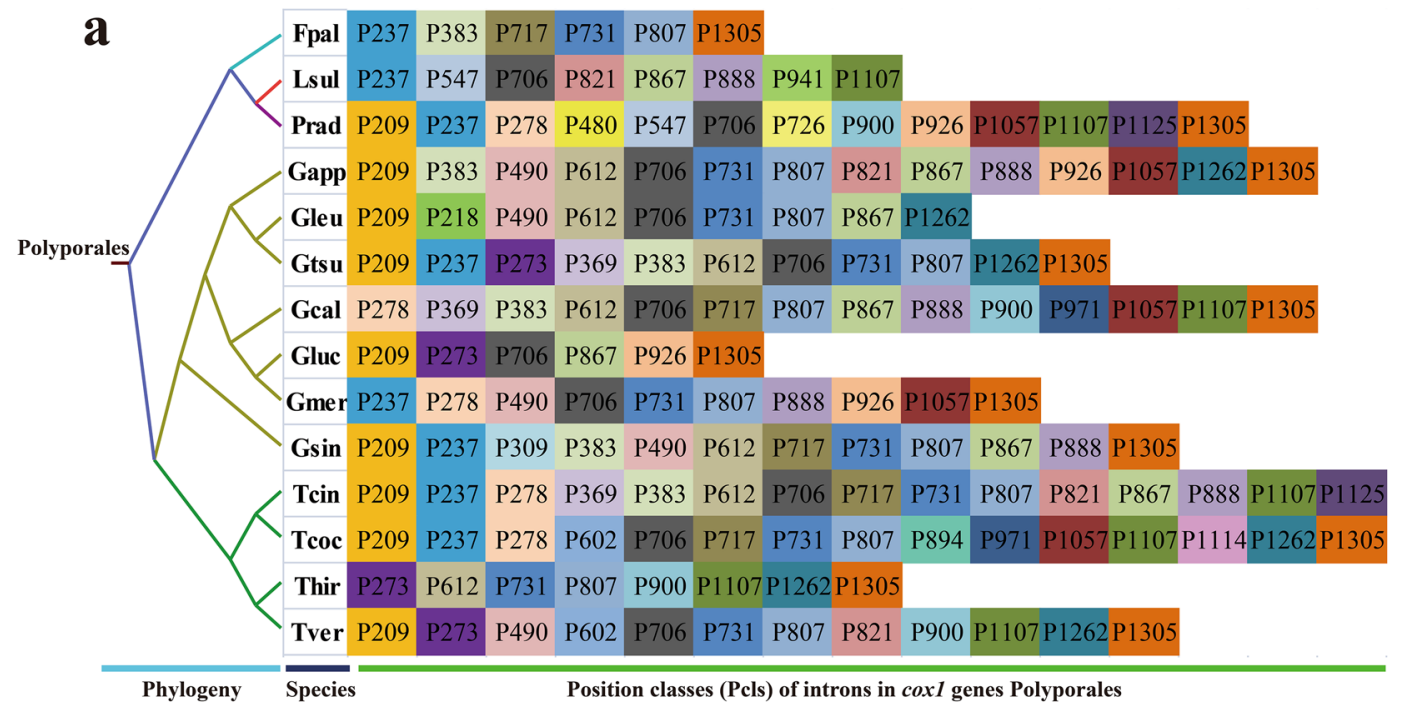

b

\begin{tabular}{|l|c|c|c|c|c|c|c|c|c|c|c|c|c|c|c|c|}
\hline Pcls & P209 & P218 & P237 & P273 & P278 & P309 & P369 & P383 & P480 & P490 & P547 & P602 & P612 & P706 & P717 & P726 \\
\hline Frequency & 9 & 1 & 8 & 4 & 5 & 1 & 3 & 6 & 1 & 5 & 2 & 2 & 7 & 11 & 5 & 1 \\
\hline Pels & P731 & P807 & P821 & P867 & P888 & P894 & P900 & P926 & P941 & P971 & P1057 P1107 & P1114 & P1125 P1262 P1305 \\
\hline Frequency & 10 & 11 & 4 & 7 & 6 & 1 & 4 & 4 & 1 & 2 & 5 & 7 & 1 & 2 & 6 & 11 \\
\hline
\end{tabular}

Figure 7. Position class (Pcl) information of coxl gene of the 14 Polyporales species. a, Pcls (orthologous introns) were named according to the insert sites (nt) in aligned reference cox1 gene (MH252535). The phylogenetic positions of the 14 Polyporales species were established using the Bayesian inference (BI) method and Maximum Likelihood (ML) method based on combined mitochondrial gene set. b, the frequency of each $\mathrm{Pcl}$ in the 14 Polyporales species.

the T. coccinea mitogenome was found to have the most introns among Trametes species. We also found a high correlation coefficient between the mitogenome size and the number of introns in Polyporales ${ }^{22,30-35}$. These findings further suggested that the dynamic change in introns is one of the main factors leading to the size variation of mitogenomes from the order Polyporales and other fungal species, which is consistent with the results of previous studies ${ }^{29,36,37}$. We also found that the mitogenome content of Trametes species varied. The four Trametes mitogenomes varied in AT and GC skews. According to the second parity rule, as long as there is no mutation or selection bias, each base in the complementary DNA strand exists at approximately equal frequencies ${ }^{38}$. The presence of AT or GC skews on the same DNA strand from different species indicated that mitogenomes of different Trametes species underwent different mutations or environmental selection ${ }^{39}$. In the present study, the core PCGs of Trametes species were found to vary frequently in length and base composition. Different core PCGs showed varied evolution rates, and some core PCGs were relatively conserved between Trametes species, whereas others showed large differentiation. All core PCGs were found to have been subjected to the pressure of purifing selection. In addition to these core PCGs, some non-conserved PCGs were also detected in Trametes species, including intronic ORFs, DNA polymerases, and RNA polymerases. Introns encoding endonucleases mainly mediate intron homing in Trametes mitogenomes ${ }^{40}$, while DNA and RNA polymerases are thought to be obtained from plasmids ${ }^{41-43}$. Several PCGs with unknown functions were also detected in Trametes species, indicating that there are still genes with unknown functions in Trametes mitogenomes that need to be revealed.

Mitochondrial gene rearrangement in Trametes species. Mitochondrial gene rearrangement was frequently detected in mitogenomes of animals, plants, and fungi ${ }^{18,44,45}$. Variations in the mitochondrial gene order can be used to infer the phylogenetic status and phylogenetic relationship of eukaryotic species ${ }^{46,47}$. The rearrangement of the animal mitogenome has been widely studied, and several models have been proposed to reveal the rearrangement of animal mitogenomes ${ }^{48,49}$. When compared with the mitogenomes of animals, the mitogenomes of fungi show greater variation in gene $\operatorname{order}^{50}$. In the present study, we detected large-scale gene rearrangements between Trametes species, including gene inversions, insertions, and migrations. Gene rearrangement events have even been observed between closely related Trametes species within the same clades, indicating that the mitochondrial gene arrangement of Trametes species is highly variable. However, the mechanism of mitochondrial gene rearrangement in fungi has not been elucidated to date. Previous studies showed that the accumulation of repetitive sequences was the main factor contributing to mitochondrial gene rearrangement in fungi ${ }^{50}$. We found a large number of repeat sequences in Trametes species, which may result in gene recombination and rearrangement in these species. 


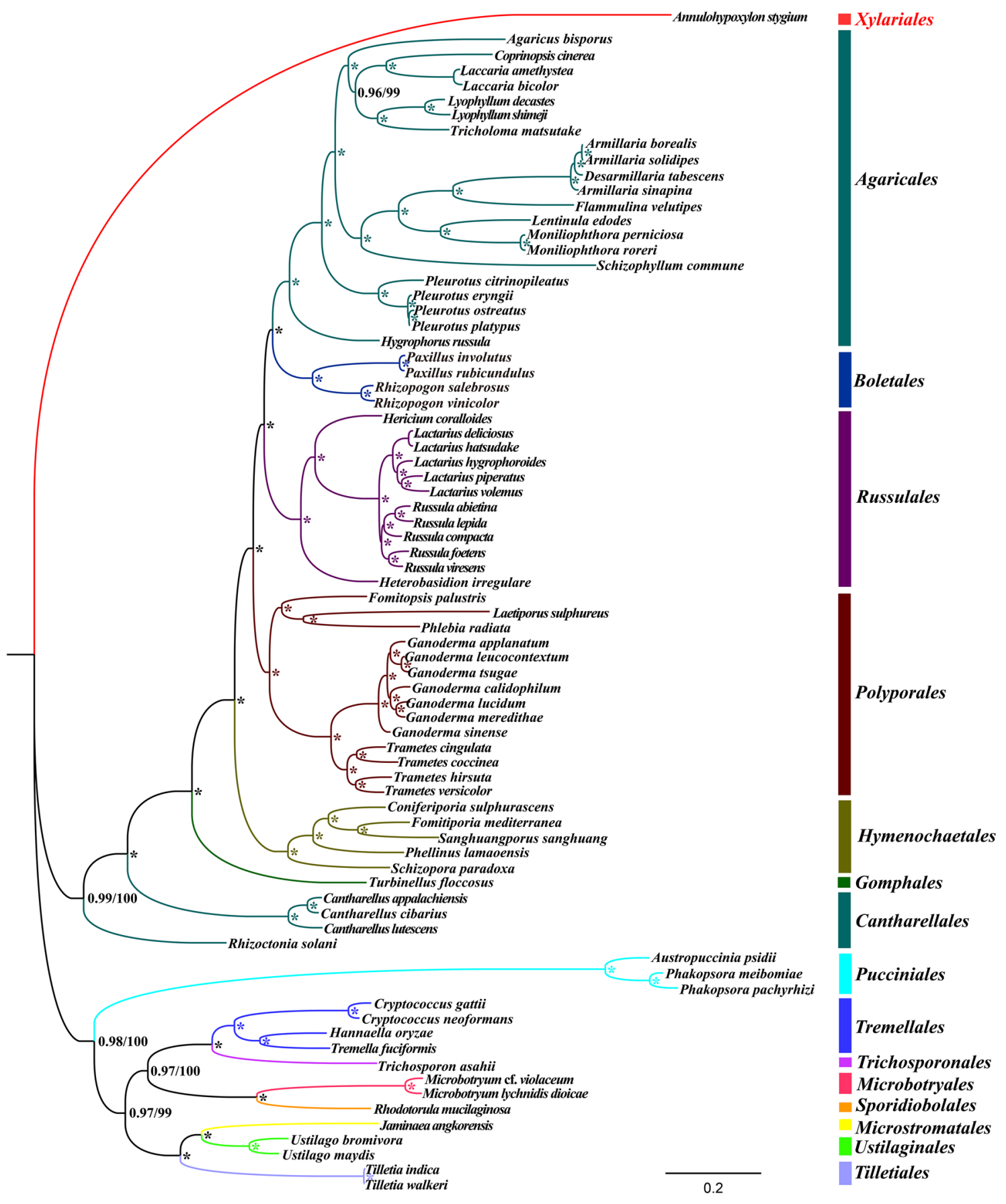

Figure 8. Phylogeny of 77 Basidiomycota species based on 15 protein coding genes and two rRNA genes using Bayesian inference (BI) and Maximum likelihood (ML) analysis. Support values are Bayesian posterior probabilities (before slash) and bootstrap (BS) values (after slash). The asterisk indicates that the BPP value is 1 and the BS value is 100 of the branch. Species and NCBI accession numbers for mitogenomes used in the phylogenetic analysis are provided in Supplementary Table S6.

Intron dynamics of Polyporales species. Introns are considered mobile genetic elements in the eukaryote mitogenome, and their dynamic changes have a significant effect on the size and organization of mitogenomes ${ }^{51-53}$. Variations in mitochondrial introns among different eukaryote lineages vary greatly. Generally, animal mitogenomes do not contain any introns, whereas plant mitogenomes mainly contain group II introns and fungal mitogenomes mainly contain group I introns $s^{51,54}$. The cox 1 gene was found to be the main host gene of introns in mitogenomes of Basidiomycetes ${ }^{55,56}$. We divided the introns of cox1 genes of 14 Polyporales species into different position classes (Pcls) according to their insertion sites in the protein coding region. The introns belonging to the same Pcl were considered homologous ${ }^{29}$. We found that the number and class of introns varied greatly between different Polyporales species, indicating that the loss/gain of introns occurred in the evolution of Polyporales. Several Pcls from common ancestors of Polyporales, such as P706, P807, and 
P1305, were found to be widely distributed in Polyporales species. However, several introns were found only in one of the 14 Polyporales species, such as P480 and P941, whereas homologous introns were detected in distant species $^{28,29}$, indicating potential intron transfer events. We also found some novel introns in Polyporales that have never been detected in other Basidiomycetes, including P218, P309, P726, P894, and P1114. The origin, evolution, and function of these novel introns need to be further analyzed.

Phylogeny of Basidiomycota species based on mitochondrial genes. Owing to the limited morphological characteristics, it is difficult to classify and identify Basidiomycetes accurately only by morphology. To date, only rRNA internal transcribed spacers $(I T S)^{57}$, RNA polymerase II subunits $(R P B)$, and elongation factor 1-a $(E F 1 a)$ genes $^{58}$ have been widely used to evaluate the phylogeny and reconstruct the early evolution of fungi. Mitochondrial genes have been widely used as molecular markers to analyze phylogenetic relationships of animals because of their unique advantages ${ }^{16,44}$. When compared with the mitogenomes of animals, the mitogenomes of fungi have been less studied, especially those of Basidiomycetes ${ }^{59}$. Currently, less than 130 complete mitogenomes of Basidiomycetes are available in the NCBI database (https://www.ncbi.nlm.nih.gov/genom e/browse\#!/overview/), which has limited comprehensive analysis of the origin and evolution of fungi. In this study, 77 Basidiomycete species were included in the phylogenic study. We have also obtained a phylogenetic tree with high support values based on the combined mitochondrial gene set, indicating that mitochondrial genes are a powerful tool for analyzing phylogenetic relationships of Basidiomycota species. With the rapid development of second and third generation sequencing technology, additional mitogenomes of fungi are needed to understand the origin and evolution of Basidiomycetes and other fungal lineages.

\begin{abstract}
Materials and methods
Assembly and annotation of Trametes mitogenomes. The raw sequencing data used for T. versicolor and T. coccinea mitogenomes assembly were downloaded from the Sequence Read Archive (SRA) database under the accession numbers SRR3927404 and SRR1588030, respectively. We conducted a series of quality control steps to generate clean reads from the raw sequencing reads, including removing adapter reads using AdapterRemoval v2 ${ }^{60}$ and filtering low-quality sequences using ngsShoRT $2.2^{61}$. We used the clean reads to assemble mitogenomes of T. versicolor and T. coccinea using SPAdes 3.9.0. $0^{62}$. Several contigs were obtained using SPAdes 3.9. $0^{62}$, and gaps between the contigs were filled using MITObim V1.9 $9^{63}$. NOVOPlasty ${ }^{64}$ was also used to assemble or test mitogenomes of the two Trametes species. After these steps, we obtained the complete mitogenomes of T. versicolor and T. coccinea, and then annotated them according to previously described methods ${ }^{33}$. We initially annotated the protein-coding genes (PCGs), open reading frames (ORFs), introns, rRNA genes, and tRNA genes in the two Trametes mitogenomes using MFannot ${ }^{65}$ and MITOS $^{66}$. PCGs or ORFs were also predicted based on the NCBI Open Reading Frame (ORF) Finder ${ }^{67}$ and annotated by BLASTP searches against the NCBI nonredundant protein sequence database ${ }^{68}$. Intron-exon borders of PCGs or ORFs were verified using Exonerate v2.2 ${ }^{69}$. We also used tRNAscan-SE v1.3.1 ${ }^{70}$ to predict or identify tRNA genes in the two Trametes mitogenomes. Finally, graphical maps of the two Trametes mitogenomes were drawn with OGDRAW $1.2^{71}$.
\end{abstract}

Mitogenome sequence analysis. Base compositions of the two Trametes mitogenomes and other Polyporales mitogenomes were analyzed using DNASTAR Lasergene v7.1 (http://www.dnastar.com/). We calculated strand asymmetries of Polyporales mitogenomes using the following formulas: AT skew $=[\mathrm{A}-\mathrm{T}] /[\mathrm{A}+\mathrm{T}]$ and GC skew $=[\mathrm{G}-\mathrm{C}] /[\mathrm{G}+\mathrm{C}]^{72}$. We analyzed codon usages within the two Trametes mitogenomes using the Sequence Manipulation Suite ${ }^{73}$. We calculated the nonsynonymous $(K a)$ and synonymous $(K s)$ substitution rates for core PCGs in the four Trametes mitogenomes using DnaSP v6.10.0174. The genetic distances between each pair of the 15 core PCGs (atp6, atp8, atp9, cob, cox1, cox2, cox3, nad1, nad2, nad3, nad4, nad4L, nad5, nad6, and rps3) were detected using MEGA v6.06 ${ }^{75}$ based on the Kimura 2-parameter (K2P) substitution model.

Repetitive element analysis. We conducted BlastN searches of the two Trametes mitogenomes against themselves ${ }^{76}$ to determine whether there were intragenomic duplications of large fragments or interspersed repeats in the two Trametes mitogenomes. We also detected tandem repeats ( $>10 \mathrm{bp}$ in length) in the two Trametes mitogenomes using a Tandem Repeats Finder ${ }^{77}$ with the default parameters. In addition, BlastN searches of the two Trametes mitogenomes were conducted against their published nuclear genomes to determine whether there were gene segments that naturally transferred between mitochondrial and nuclear genomes ${ }^{78,79}$.

Comparative mitogenomic analysis and intron analysis. The genome sizes, GC contents, base compositions, start and stop codons, gene numbers, and intron numbers of Polyporales mitogenomes were compared to identify variations and similarities between them. Introns of cox 1 genes in the 15 Polyporales mitogenomes that have been published to date were classified into different position classes (Pcls) according to the method described by Ferandon et al. ${ }^{80}$. The cox 1 genes of 15 Polyporales mitogenomes were first aligned with the cox 1 gene of the medical fungus Ganoderma calidophilum ${ }^{22}$ using Clustal $\mathrm{W}^{81}$. Each Pcl was composed of introns inserted at the same position of the cox 1 reference gene based on the insert sites (nt) in the corresponding reference gene. Identical Pcls were considered orthologous introns with high sequence similarity.

Phylogenetic analysis. A phylogenetic tree of 77 Basidiomycota species was constructed to investigate the status of Trametes species in the phylum Basidiomycota using previously described methods ${ }^{33,82}$ based on the combined mitochondrial gene set (15 core PCGs). We used Annulohypoxylon stygium from the phylum Ascomycota as the outgroup ${ }^{83}$. Individual mitochondrial genes were first aligned using the MAFFT v7.037 software ${ }^{84}$, 
after which we concatenated the aligned mitochondrial genes into a combined mitochondrial gene set using the SequenceMatrix v1.7.8 software ${ }^{85}$. A preliminary partition homogeneity test was then conducted to detect potential phylogenetic conflicts between different genes. Best-fit models of partitioning schemes and evolution for the combined mitochondrial gene set were determined based on the PartitionFinder 2.1.1 software ${ }^{86}$. We constructed phylogenetic trees using both Bayesian inference (BI) and maximum likelihood (ML) methods, with MrBayes v3.2.6 ${ }^{87}$ used for the BI analysis and RAxML v 8.0.0 88 for the ML analysis.

Data availability. The complete mitogenomes of T. versicolor and T. coccinea were deposited in the GenBank database under accession numbers MT479165 and MT479166, respectively.

Received: 9 June 2020; Accepted: 12 January 2021

Published online: 28 January 2021

\section{References}

1. Olou, B. A., Krah, F. S., Piepenbring, M., Yorou, N. S. \& Langer, E. Diversity of Trametes (Polyporales, Basidiomycota) in tropical Benin and description of new species Trametes parvispora. MycoKeys 65, 25-47 (2020).

2. Yamashita, S., Hattori, T. \& Abe, H. Host preference and species richness of wood-inhabiting aphyllophoraceous fungi in a cool temperate area of Japan. Mycologia 102, 11-19 (2010).

3. Adekunle, A. E. et al. Chitosan multiple addition enhances laccase production from Trametes versicolor. Bioprocess. Biosyst. Eng. 38, 1973-1981 (2015).

4. Patil, P. D. \& Yadav, G. D. Comparative studies of white-rot fungal strains (trametes hirsuta MTCC-1171 and phanerochaete chrysosporium NCIM-1106) for effective degradation and bioconversion of ferulic acid. ACS Omega 3, 14858-14868 (2018).

5. Pinheiro, V. E., Michelin, M., Vici, A. C., de Almeida, P. Z. \& Teixeira de Moraes Polizeli, M. L. Trametes versicolor laccase production using agricultural wastes: a comparative study in Erlenmeyer flasks, bioreactor and tray. Bioprocess. Biosyst. Eng. 43, 507-514 (2020).

6. Zhang, H. et al. Characterization of a manganese peroxidase from white-rot fungus Trametes sp.48424 with strong ability of degrading different types of dyes and polycyclic aromatic hydrocarbons. J. Hazard. Mater. 320, 265-277 (2016).

7. Gao, X. et al. Proteomic analysis reveals large amounts of decomposition enzymes and major metabolic pathways involved in algicidal process of Trametes versicolor F21a. Sci. Rep. 7, 3907 (2017).

8. Rekik, H. et al. Physical and enzymatic properties of a new manganese peroxidase from the white-rot fungus Trametes pubescens strain i8 for lignin biodegradation and textile-dyes biodecolorization. Int. J. Biol. Macromol. 125, 514-525 (2019).

9. Zheng, F. et al. A novel laccase from white rot fungus Trametes orientalis: purification, characterization, and application. Int. J. Biol. Macromol. 102, 758-770 (2017).

10. Haridas, S. \& Gantt, J. S. The mitochondrial genome of the wood-degrading basidiomycete Trametes cingulata. FEMS Microbiol Lett 308, 29-34 (2010).

11. Pavlov, A. R. et al. Draft genome sequence of the fungus Trametes hirsuta 072. Genome. Announc. 3, e01287-15 (2015).

12. Lang, B. F., Gray, M. W. \& Burger, G. Mitochondrial genome evolution and the origin of eukaryotes. Annu. Rev. Genet. 33, 351-397 (1999).

13. Munoz-Gomez, S. A., Wideman, J. G., Roger, A. J. \& Slamovits, C. H. The origin of mitochondrial cristae from alphaproteobacteria. Mol. Biol. Evol. 34, 943-956 (2017).

14. Viscomi, C. \& Zeviani, M. Strategies for fighting mitochondrial diseases. J. Intern. Med. 287, 665-684 (2020).

15. Gorman, G. S. et al. Prevalence of nuclear and mitochondrial DNA mutations related to adult mitochondrial disease. Ann. Neurol. 77, 753-759 (2015).

16. Cameron, S. L. Insect mitochondrial genomics: implications for evolution and phylogeny. Annu. Rev. Entomol. 59, 95-117 (2014).

17. Li, Q. et al. Characterization of the mitochondrial genomes of three species in the ectomycorrhizal genus Cantharellus and phylogeny of Agaricomycetes. Int. J. Biol. Macromol. 118, 756-769 (2018).

18. Li, Q. et al. Comparative mitogenomics reveals large-scale gene rearrangements in the mitochondrial genome of two Pleurotus species. Appl. Microbiol. Biotechnol. 102, 6143-6153 (2018).

19. Zhang, Y. et al. Phylogeny, evolution and mitochondrial gene order rearrangement in scale worms (Aphroditiformia, Annelida). Mol. Phylogenet. Evol. 125, 220-231 (2018).

20. Yang, H. et al. Characterization of the complete mitochondrial genome sequences of three croakers (perciformes, sciaenidae) and novel insights into the phylogenetics. Int. J. Mol. Sci. 19, 1741 (2018).

21. Li, Q. et al. Comparative mitogenome analysis reveals mitochondrial genome differentiation in ectomycorrhizal and asymbiotic Amanita species. Front. Microbiol. 11, 1382 (2020).

22. Li, Q. et al. The complete mitochondrial genomes of five important medicinal Ganoderma species: features, evolution, and phylogeny. Int. J. Biol. Macromol. 139, 397-408 (2019).

23. Li, Q. et al. Characterization and comparison of the mitochondrial genomes from two Lyophyllum fungal species and insights into phylogeny of Agaricomycetes. Int. J. Biol. Macromol. 121, 364-372 (2019).

24. Wang, X. et al. The $256 \mathrm{~kb}$ mitochondrial genome of Clavaria fumosa is the largest among phylum Basidiomycota and is rich in introns and intronic ORFs. Ima Fungus 11, 26 (2020).

25. Darling, A. C., Mau, B., Blattner, F. R. \& Perna, N. T. Mauve: multiple alignment of conserved genomic sequence with rearrangements. Genome Res. 14, 1394-1403 (2004).

26. Ye, J., Cheng, J., Ren, Y., Liao, W. \& Li, Q. The first mitochondrial genome for geastrales (Sphaerobolus stellatus) reveals intron dynamics and large-scale gene rearrangements of basidiomycota. Front. Microbiol. 11, 1970 (2020).

27. Wang, X. et al. The $206 \mathrm{kbp}$ mitochondrial genome of Phanerochaete carnosa reveals dynamics of introns, accumulation of repeat sequences and plasmid-derived genes. Int. J. Biol. Macromol. 162, 209-219 (2020).

28. Kolesnikova, A. I. et al. Mobile genetic elements explain size variation in the mitochondrial genomes of four closely-related Armillaria species. BMC Genom. 20, 351 (2019).

29. Ferandon, C. et al. The agaricus bisporus cox 1 gene: the longest mitochondrial gene and the largest reservoir of Mitochondrial Group I Introns. PLoS ONE 5, e14048 (2010).

30. Salavirta, H. et al. Mitochondrial genome of Phlebia radiata is the second largest (156 kbp) among fungi and features signs of genome flexibility and recent recombination events. PLOS ONE 9, e97141 (2014).

31. Tanaka, Y. et al. The complete genome sequence and phylogenetic analysis of the mitochondrial DNA of the wood-decaying fungus Fomitopsis palustris. Genes Genom. 39, 1377-1385 (2017).

32. Li, J. et al. Complete mitochondrial genome of the medicinal mushroom Ganoderma lucidum. PLoS ONE 8, e72038 (2013). 
33. Li, Q. et al. Characterization and phylogenetic analysis of the complete mitochondrial genome of the medicinal fungus Laetiporus sulphureus. Sci. Rep. 8, 9104 (2018).

34. Wang, X. C., Shao, J. J. \& Liu, C. The complete mitochondrial genome of the medicinal fungus Ganoderma applanatum (Polyporales, Basidiomycota). Mitochondrial DNA Part A 27, 2813-2814 (2016).

35. Wang, X. C. et al. The complete mitochondrial genome of the white-rot fungus Ganoderma meredithiae (Polyporales, Basidiomycota). Mitochondrial DNA Part A 27, 4197-4198 (2016).

36. Himmelstrand, K., Olson, A., Brandstrom Durling, M., Karlsson, M. \& Stenlid, J. Intronic and plasmid-derived regions contribute to the large mitochondrial genome sizes of Agaricomycetes. Curr. Genet. 60, 303-313 (2014).

37. Liu, W. et al. The mitochondrial genome of Morchella importuna $(272.2 \mathrm{~kb})$ is the largest among fungi and contains numerous introns, mitochondrial non-conserved open reading frames and repetitive sequences. Int. J. Biol. Macromol. 143, 373-381 (2019).

38. Chen, H., Sun, S., Norenburg, J. L. \& Sundberg, P. Mutation and selection cause codon usage and bias in mitochondrial genomes of ribbon worms (Nemertea). PLoS ONE 9, e85631 (2014).

39. Chen, C. et al. Characterization of the mitochondrial genome of the pathogenic fungus Scytalidium auriculariicola (Leotiomycetes) and insights into its phylogenetics. Sci. Rep. 9, 17447 (2019).

40. Chi, S. I., Urbarova, I. \& Johansen, S. D. Expression of homing endonuclease gene and insertion-like element in sea anemone mitochondrial genomes: lesson learned from Anemonia viridis. Gene 652, 78-86 (2018).

41. Cahan, P. \& Kennell, J. C. Identification and distribution of sequences having similarity to mitochondrial plasmids in mitochondrial genomes of filamentous fungi. Mol. Genet. Genom. 273, 462-473 (2005).

42. Formighieri, E. F. et al. The mitochondrial genome of the phytopathogenic basidiomycete Moniliophthora perniciosa is $109 \mathrm{~kb}$ in size and contains a stable integrated plasmid. Mycol. Res. 112, 1136-1152 (2008).

43. Wu, P. et al. The mitogenomes of two saprophytic Boletales species (Coniophora) reveals intron dynamics and accumulation of plasmid-derived and non-conserved genes. Comput. Struct. Biotechnol. J. 19, 401-414 (2021).

44. Boore, J. L. Animal mitochondrial genomes. Nucleic Acids Res. 27, 1767-1780 (1999).

45. Li, Q. et al. Comparative mitochondrial genome analysis of two ectomycorrhizal fungi (Rhizopogon) reveals dynamic changes of intron and phylogenetic relationships of the subphylum Agaricomycotina. Int. J. Mol. Sci. 20, 5167 (2019).

46. Sankoff, D. et al. Gene order comparisons for phylogenetic inference: evolution of the mitochondrial genome. Proc. Natl. Acad. Sci. U. S. A. 89, 6575-6579 (1992).

47. Li, Q. et al. Comparative mitogenome analysis of two ectomycorrhizal fungi (Paxillus) reveals gene rearrangement, intron dynamics, and phylogeny of basidiomycetes. IMA Fungus 11, 12 (2020).

48. Lavrov, D. V., Boore, J. L. \& Brown, W. M. Complete mtDNA sequences of two millipedes suggest a new model for mitochondrial gene rearrangements: duplication and nonrandom loss. Mol. Biol. Evol. 19, 163-169 (2002).

49. Xia, Y., Zheng, Y., Murphy, R. W. \& Zeng, X. Intraspecific rearrangement of mitochondrial genome suggests the prevalence of the tandem duplication-random loss (TDLR) mechanism in Quasipaa boulengeri. BMC Genom. 17, 965 (2016).

50. Aguileta, G. et al. High variability of mitochondrial gene order among fungi. Genome Biol. Evol. 6, 451-465 (2014).

51. Repar, J. \& Warnecke, T. Mobile introns shape the genetic diversity of their host genes. Genetics 205, 1641-1648 (2017).

52. Burke, J. M. Molecular genetics of group I introns: RNA structures and protein factors required for splicing-a review. Gene 73, 273-294 (1988).

53. Hamari, Z., Juhasz, A. \& Kevei, F. Role of mobile introns in mitochondrial genome diversity of fungi (a mini review). Acta Microbiol. Immunol. Hung. 49, 331-335 (2002).

54. Fan, W. W., Zhang, S. \& Zhang, Y. J. The complete mitochondrial genome of the Chan-hua fungus Isaria cicadae: a tale of intron evolution in Cordycipitaceae. Environ. Microbiol. 21, 864-879 (2019).

55. Li, Q. et al. The complete mitochondrial genomes of two model ectomycorrhizal fungi (Laccaria): features, intron dynamics and phylogenetic implications. Int. J. Biol. Macromol. 145, 974-984 (2020).

56. Wang, X. et al. The complete mitochondrial genome of medicinal fungus Taiwanofungus camphoratus reveals gene rearrangements and intron dynamics of Polyporales. Sci. Rep. 10, 16500 (2020).

57. Schoch, C. L. et al. Nuclear ribosomal internal transcribed spacer (ITS) region as a universal DNA barcode marker for Fungi. Proc. Natl. Acad. Sci. U. S. A. 109, 6241-6246 (2012).

58. James, T. Y. et al. Reconstructing the early evolution of Fungi using a six-gene phylogeny. Nature 443, 818-822 (2006).

59. Wang, Y. \& Xu, J. Mitochondrial genome polymorphisms in the human pathogenic fungus Cryptococcus neoformans. Front. Microbiol. 11, 706 (2020).

60. Schubert, M., Lindgreen, S. \& Orlando, L. AdapterRemoval v2: rapid adapter trimming, identification, and read merging. BMC Res. Notes 9,88 (2016).

61. Chen, C., Khaleel, S. S., Huang, H. \& Wu, C. H. Software for pre-processing Illumina next-generation sequencing short read sequences. Sour. Code Biol. Med. 9 , 8 (2014).

62. Bankevich, A. et al. SPAdes: a new genome assembly algorithm and its applications to single-cell sequencing. J. Comput. Biol. 19, 455-477 (2012).

63. Hahn, C., Bachmann, L. \& Chevreux, B. Reconstructing mitochondrial genomes directly from genomic next-generation sequencing reads-a baiting and iterative mapping approach. Nucleic Acids Res. 41, e129 (2013).

64. Dierckxsens, N., Mardulyn, P. \& Smits, G. NOVOPlasty: de novo assembly of organelle genomes from whole genome data. Nucleic Acids Res. 45, e18 (2017).

65. Valach, M., Burger, G., Gray, M. W. \& Lang, B. F. Widespread occurrence of organelle genome-encoded 5S rRNAs including permuted molecules. Nucleic Acids Res. 42, 13764-13777 (2014).

66. Bernt, M. et al. MITOS: improved de novo metazoan mitochondrial genome annotation. Mol. Phylogenet. Evol. 69, 313-319 (2013).

67. Coordinators, N. R. Database resources of the National Center for Biotechnology Information. Nucleic Acids Res. 45, D12-D17 (2017).

68. Bleasby, A. J. \& Wootton, J. C. Construction of validated, non-redundant composite protein sequence databases. Protein Eng. 3, $153-159$ (1990).

69. Slater, G. S. \& Birney, E. Automated generation of heuristics for biological sequence comparison. BMC Bioinform. 6, 31 (2005).

70. Lowe, T. M. \& Chan, P. P. tRNAscan-SE On-line: integrating search and context for analysis of transfer RNA genes. Nucleic Acids Res. 44, W54-W57 (2016).

71. Lohse, M., Drechsel, O., Kahlau, S. \& Bock, R. OrganellarGenomeDRAW-a suite of tools for generating physical maps of plastid and mitochondrial genomes and visualizing expression data sets. Nucleic Acids Res. 41, W575-W581 (2013).

72. Wang, J. et al. Comparative mitogenomic analysis of mirid bugs (Hemiptera: Miridae) and evaluation of potential DNA barcoding markers. PeerJ 5, e3661 (2017).

73. Stothard, P. The sequence manipulation suite: JavaScript programs for analyzing and formatting protein and DNA sequences. Biotechniques 28, 1102-1104 (2000).

74. Rozas, J. et al. DnaSP 6: DNA sequence polymorphism analysis of large data sets. Mol. Biol. Evol. 34, 3299-3302 (2017).

75. Caspermeyer, J. MEGA evolutionary software re-engineered to handle today's big data demands. Mol. Biol. Evol. 33, 1887 (2016).

76. Chen, Y., Ye, W., Zhang, Y. \& Xu, Y. High speed BLASTN: an accelerated MegaBLAST search tool. Nucleic Acids Res. 43, 7762-7768 (2015).

77. Benson, G. Tandem repeats finder: a program to analyze DNA sequences. Nucleic Acids Res. 27, 573-580 (1999). 
78. Couturier, M. et al. Enhanced degradation of softwood versus hardwood by the white-rot fungus Pycnoporus coccineus. Biotechnol. Biofuels 8, 216 (2015).

79. Floudas, D. et al. The paleozoic origin of enzymatic lignin decomposition reconstructed from 31 fungal genomes. Science 336, 1715-1719 (2012).

80. Ferandon, C. et al. The Agaricus bisporus coxl gene: the longest mitochondrial gene and the largest reservoir of mitochondrial group i introns. PLoS ONE 5, e14048 (2010).

81. Thompson, J. D., Higgins, D. G. \& Gibson, T. J. CLUSTAL W: improving the sensitivity of progressive multiple sequence alignment through sequence weighting, position-specific gap penalties and weight matrix choice. Nucleic Acids Res. 22, 4673-4680 (1994).

82. Li, Q. et al. The first eleven mitochondrial genomes from the ectomycorrhizal fungal genus (Boletus) reveal intron loss and gene rearrangement. Int. J. Biol. Macromol. https://doi.org/10.1016/j.ijbiomac.2021.01.087 (2021).

83. Deng, Y. et al. Comparison of the mitochondrial genome sequences of six annulohypoxylon stygium isolates suggests short fragment insertions as a potential factor leading to larger genomic size. Front. Microbiol. 9, 2079 (2018).

84. Katoh, K., Rozewicki, J. \& Yamada, K. D. MAFFT online service: multiple sequence alignment, interactive sequence choice and visualization. Brief Bioinform. 20, 1160-1166 (2019).

85. Vaidya, G., Lohman, D. L. \& Meier, R. SequenceMatrix: concatenation software for the fast assembly of multi-gene datasets with character set and codon information. Cladistics 27, 171-180 (2011).

86. Lanfear, R., Frandsen, P. B., Wright, A. M., Senfeld, T. \& Calcott, B. PartitionFinder 2: new methods for selecting partitioned models of evolution for molecular and morphological phylogenetic analyses. Mol. Biol. Evol. 34, 772-773 (2017).

87. Ronquist, F. et al. MrBayes 3.2: efficient Bayesian phylogenetic inference and model choice across a large model space. Syst. Biol. 61, 539-542 (2012).

88. Stamatakis, A. RAxML version 8: a tool for phylogenetic analysis and post-analysis of large phylogenies. Bioinformatics 30, 1312$1313(2014)$.

\section{Acknowledgments}

We thank LetPub (www.letpub.com) for its linguistic assistance during the preparation of this manuscript.

\section{Author contributions}

Conceived and designed experiments: C.C., Q.L. and D.L. Performed the experiments: C.C., Q.L., R.F and J.W.. Analyzed the data: C.C., Q.L., G.D. and D.L. Contributed reagents/materials/analysis tools: C.C., Q.L., R.F., J.W., J.C. and D.L. Wrote the paper: C.C.

\section{Funding}

This research was funded by the Foundation Program of the Financial \& Innovational Capacity Building Project of Sichuan (2019LWJJ-007\&2016GYSH-014).

\section{Competing interests}

The authors declare no competing interests.

\section{Additional information}

Supplementary Information The online version contains supplementary material available at https://doi. org/10.1038/s41598-021-82040-7.

Correspondence and requests for materials should be addressed to D.L.

Reprints and permissions information is available at www.nature.com/reprints.

Publisher's note Springer Nature remains neutral with regard to jurisdictional claims in published maps and institutional affiliations.

(c) (i) Open Access This article is licensed under a Creative Commons Attribution 4.0 International License, which permits use, sharing, adaptation, distribution and reproduction in any medium or format, as long as you give appropriate credit to the original author(s) and the source, provide a link to the Creative Commons licence, and indicate if changes were made. The images or other third party material in this article are included in the article's Creative Commons licence, unless indicated otherwise in a credit line to the material. If material is not included in the article's Creative Commons licence and your intended use is not permitted by statutory regulation or exceeds the permitted use, you will need to obtain permission directly from the copyright holder. To view a copy of this licence, visit http://creativecommons.org/licenses/by/4.0/.

(c) The Author(s) 2021 